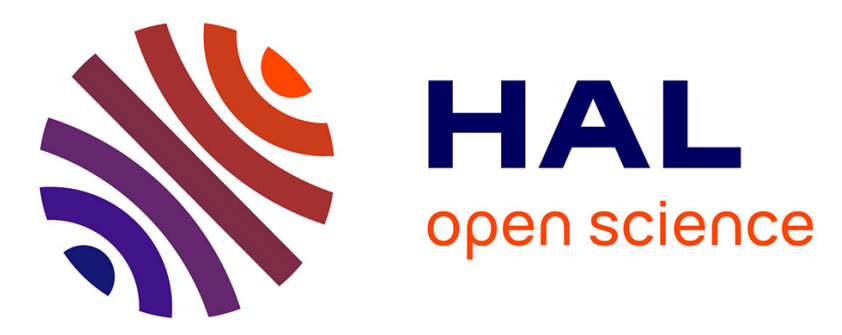

\title{
SERS active systems of water-soluble polythiophene and plasmonic nanoparticles: preparation and optical properties
}

\author{
S. Kazim, J. Pfleger, K. Halašová, M. Procházka, D. Bondarev, J. Vohlídal
}

\section{- To cite this version:}

S. Kazim, J. Pfleger, K. Halašová, M. Procházka, D. Bondarev, et al.. SERS active systems of watersoluble polythiophene and plasmonic nanoparticles: preparation and optical properties. European Physical Journal: Applied Physics, 2011, 55 (2), 10.1051/epjap/2011100417 . hal-00723594

\author{
HAL Id: hal-00723594 \\ https://hal.science/hal-00723594
}

Submitted on 11 Aug 2012

HAL is a multi-disciplinary open access archive for the deposit and dissemination of scientific research documents, whether they are published or not. The documents may come from teaching and research institutions in France or abroad, or from public or private research centers.
L'archive ouverte pluridisciplinaire HAL, est destinée au dépôt et à la diffusion de documents scientifiques de niveau recherche, publiés ou non, émanant des établissements d'enseignement et de recherche français ou étrangers, des laboratoires publics ou privés. 


\title{
SERS Active Systems of Water Soluble Polythiophene and Plasmonic Nanoparticles: Preparation and Optical Properties
}

\author{
Samrana Kazim¹, Jiří Pfleger ${ }^{1}$ *, Klára Halašová ${ }^{1}$, Marek Procházka², Dmitrij \\ Bondarev $^{3}$, Jiří Vohlídal ${ }^{3}$ \\ ${ }^{1}$ Institute of Macromolecular Chemistry, Academy of Sciences of the Czech Republic, v.v.i., Heyrovsky \\ Sq. 2, 16206 Prague 6, Czech Republic \\ ${ }^{2}$ Charles University in Prague, Faculty of Mathematics and Physics, Institute of Physics, Ke Karlovu \\ 5, 12116 Prague 2, Czech Republic \\ ${ }^{3}$ Charles University in Prague, Faculty of Science, Department of Physical and Macromolecular \\ Chemistry, Hlavova 2030, 12840 Prague 2, Czech Republic \\ * Corresponding author: Dept. of Polymer Materials, Institute of Macromolecular Chemistry ASCR, \\ v.v.i., Heyrovsky Sq. 2, 16206 Prague 6, Czech Republic. Tel.: +420222511696, Fax: \\ +420222516969. Email address: pfleger@imc.cas.cz
}

\section{SERS Active Systems with Water Soluble Polythiophene}

\begin{abstract}
Aggregated systems of silver (Ag) and gold (Au) nanoparticles coated with cationic polythiophene were prepared, which showed optical properties typical for strongly coupled plasmonic excitations. The procedure allowed tune up the 3-dimensional arrangement of nanoparticles assembly for achieving strong SERS effects at the excitation wavelength depending on the polymer concentration. By combination with an anionic derivative of polythiophene thin multilayer films composed of alternating anionic and cationic polythiophene with mutually interacting plasmonic $\mathrm{Au}$ nanoparticles were successfully prepared using layer-by-layer deposition.
\end{abstract}

\section{Introduction}

Hybrid nanocomposite materials containing plasmonic metal nanoparticles (NPs) embedded in a polymer matrix have emerged recently as a boosting area of research. Besides increased electrical conductivity due to the metallic character of the filler such nanocomposites were suggested to work as photochemical catalysts [1], optical sensors [2,3], SERS probes [4,5] or nonvolatile rewritable memories [6]. When sized in a range $5-100 \mathrm{~nm}$ the metal nanoparticles possess optical properties that stem from plasmons - collective oscillations of free electrons excited by incident light of a proper wavelength. Such oscillations have a resonance frequency that can be tuned by their size, shape, aspect ratio, dielectric constant of the environment and their mutual arrangement in 3-dimensional assemblies. For Ag and Au nanoparticles the resonance falls within the visible spectral region and it is accompanied by a local enhancement of the optical electric field in a close vicinity of the nanoparticle surface. The enhancement of incident light and of light scattered nonelastically by molecules adsorbed on the nanoparticle surface is the principle of the surface enhanced Raman scattering (SERS) or surface enhanced resonance Raman scattering (SERRS). Large enhancements of SERS signal of the order of $10^{14}$ were reported on systems that contain "hot spots" formed in properly assembled nanoparticles in dimers or aggregates $[7,8,9]$.

The combination of plasmonic properties of metal nanoparticles with semiconductive properties of $\pi$-conjugated polymers in composite materials brings a promising functionality 
for advanced optoelectronic applications due to the synergy effect of surface plasmons and delocalized $\pi$-electrons in the polymer backbone. Superquenching of fluorescence [10] or localization of optical field in a donor-acceptor interface located near the nanoparticle surface, leading to better harvesting of absorbed light for photovoltaic energy conversion [11], are the examples of such phenomena. The effect of metal nanoparticles on the $\pi-\pi^{*}$ absorption band of conjugated polymers and the effect of delocalized $\pi$-electron system of semiconductive polymers on the electronic coupling between NPs are a hot subject of debate. Understanding and tuning such effects could lead to hybrid optical devices with improved optical properties.

Metal nanoparticles are mostly synthesized by reduction of metal salts in water environment as hydrosols stabilized only by electrostatic forces. The insolubility of semiconductive polymers in water miscible solvents brings major obstacle for the preparation of plasmonic nanocomposites in a form of inks suitable for printable electronics and photonics. For mixing nanoparticles with polymer solutions they need to be stabilized with hydrophobic molecules. Since the enhancement of optical field decreases rapidly, as $r^{-6}$, with the distance $r$ from the nanoparticle surface such steric stabilization hinders an efficient interaction between plasmons and conjugated polymer.

$\pi$-conjugated polyelectrolytes are polymers that combine the semiconductive properties of the hydrophobic conjugated polymer backbone with ionic character of the side-groups, which yield the polymer solubility in water and water miscible solvents and also allow interactions with other charged species in the system. Since metal nanoparticles are usually negatively charged due to the presence of residual ions from their synthesis, conjugated polyelectrolyte can easily adsorb at the nanoparticle surface. Combining polymers with oppositely charged ionic groups multilayer structures can be prepared using layer-by-layer deposition technique.

Recently, we showed that a water soluble derivative of polyacetylene containing cationic pyridinium groups can serve as a suitable matrix for the Ag nanoparticles [12]. Moreover, due to the electrostatic interactions the aggregation of the nanoparticles can be controlled to form fractal aggregates with "hot spots" of extreme enhancement of optical field. In this paper we employed similar approach for the preparation of nanostructured plasmonic materials based on water soluble cationic derivative of polythiophene.

\section{Experimental Section}

\subsection{Materials:}

Regioregular poly\{3-[6-(1-methylimidazolium-3-yl)hexyl]thiophene-2,5-diyl bromide\}, PMHT-Br, was synthesized using recently developed two step procedure [13]: first the regioregular poly[3-(6-bromohexyl)thiophene-2,5-diyl] was prepared from 3-(6bromohexyl)thiophene by Kumada coupling reaction catalyzed with Ni based catalyst, which was subsequently modified using reaction with 1-methylimidazole. The resulting cationic polythiophene polyelectrolyte is soluble in water, common polar solvents like dimethyl sulfoxide (DMSO) or dimethylformamide and some alcohols.

Poly [(thiophene-3-yl)acetic acid], PTAA, was synthesized according to the reference [14]. Chemical structures of both cationic polythiophene, PMHT-Br, and anionic polythiophene, PTAA, are shown in Figure 1.

Silver colloid containing borate-stabilized Ag nanoparticles was prepared by reduction of $\mathrm{AgNO}_{3}$ with $\mathrm{NaBH}_{4}$ according to the procedure described in refs $[12,15,16]$. Briefly, $3.5 \mathrm{mg}$ of $\mathrm{NaBH}_{4}$ was dissolved in $75 \mathrm{ml}$ of deionized water and cooled to $2{ }^{\circ} \mathrm{C}$. After reaching this temperature, $9 \mathrm{~mL}$ of $2.2 \cdot 10^{-3} \mathrm{M}$ aqueous solution of $\mathrm{AgNO}_{3}$ (pre cooled down to $\sim 8^{\circ} \mathrm{C}$ ) was dropwise added under vigorous stirring. Stirring was continued for $45 \mathrm{~min}$. As a result, the yellowish sol was obtained that was aged for several weeks before being used in further experiments. 
Gold colloid containing citrate stabilized gold nanoparticles (Au NPs) was prepared by the citrate reduction of $\mathrm{HAuCl}_{4}$ according to the procedure of Lee and Meisel [17]: $125 \mathrm{ml}$ of 1.2 $\mathrm{mM}$ solution of $\mathrm{HAuCl}_{4}$ was brought to boiling under vigorous stirring. To this solution 12.5 $\mathrm{ml}$ of $1 \%$ sodium citrate solution was added rapidly. The reaction mixture quickly changed the color from pale yellow to dark red. The boiling continued for $10 \mathrm{~min}$ and then the hydrosol was cooled down under stirring for another $45 \mathrm{~min}$. Concentration of silver and gold in the resulting hydrosols was $2.4 \cdot 10^{-4} \mathrm{M}$ and $1.1 \cdot 10^{-3} \mathrm{M}$, respectively.

$\mathrm{Ag}-\mathrm{NPS} / \mathrm{PMHT}-\mathrm{Br}$ and $\mathrm{Au}-\mathrm{NP}$ /PMHT-Br sols for UV-Vis and SERS spectroscopy were prepared by adding a measured volume of the stock solution of PMHT-Br $\left(1 \cdot 10^{-1} \mathrm{M}\right.$ of monomer units in DMSO) to $2 \mathrm{~mL}$ of $\mathrm{Ag}$ or Au hydrosol, respectively. Molar concentrations of PMHT-Br in resulting sols, $c_{\text {pol }}$, were set to be: $5 \cdot 10^{-8} \mathrm{M}, 5 \cdot 10^{-7} \mathrm{M}, 5 \cdot 10^{-6} \mathrm{M}, 1 \cdot 10^{-6} \mathrm{M}$, $1 \cdot 10^{-5} \mathrm{M}$ and $1 \cdot 10^{-4} \mathrm{M}$. The same procedure was adopted for the preparation of sols with anionic polythiophene PTAA.

PMHT-Br/Au-NPs/PTAA multilayered structures were prepared using the layer-by-layer deposition technique. A solid substrate (quartz, mica, ITO) was consecutively immersed into aqueous solutions of the cationic PMHT-Br, Au hydrosol and the anionic PTAA, each for 10 min. The concentration of polymer solutions was $10^{-5} \mathrm{M}$. Between subsequent immersions the samples were washed by water to remove the polymer not attached by electrostatic forces.

\subsection{Instrumentation}

$U V$-Vis absorption spectra were recorded in the spectral range $200-800 \mathrm{~nm}$ using a double beam Perkin Elmer Lambda $950 \mathrm{UV}$-Vis spectrophotometer. The sols were measured in a $1 \mathrm{~cm}$ quartz cuvette, multilayered thin films deposited on quartz substrates were placed in a solid sample holder during the measurement.

TEM images of Ag and Au colloids were obtained with a JEOL JEM 200 CX transmission electron microscope. The samples were prepared by depositing a small drop of the sol onto a copper-mesh grid covered by a carbon foil and left to dry on air.

SERS spectra of Ag/PMHT-Br and Au/PMHT-Br systems were measured from solutions placed in a $1 \mathrm{~cm}$ five-window quartz cuvette at room temperature. Spectra were recorded with a Raman spectrometer equipped with a monochromator (1800 gr/mm grating, Jobin-YvonSpex 270M) and a CCD detector (100 x 1340 pixels, Princeton Instruments) in a right angle scattering geometry. The excitation with $514.5 \mathrm{~nm}$ line (argon laser Innova 300) was used for the $\mathrm{Ag} / \mathrm{PMHT}-\mathrm{Br}$ sols, whereas the $\mathrm{Au} / \mathrm{PMHT}-\mathrm{Br}$ sols were excited using $647.1 \mathrm{~nm}$ line $\left(\mathrm{Kr}^{+}\right.$ ion laser, Melles Griot). The spectra were accumulated for $150 \mathrm{~s}$ for each measurement. Necessary post-acquisition spectra treatment was done by the SPECTRACALC program (Galactic Industries Corp.). A baseline correction, normalization (the $618 \mathrm{~cm}^{-1}$ DMSO band was used as an internal intensity standard) and quantitative analysis of Raman spectra were carried out using factor analysis.

\section{Results and Discussion}

TEM images of Ag and Au nanoparticles as deposited from corresponding colloids on carbon coated grids are presented in Figs. $2 \mathrm{a}$ and $2 \mathrm{~b}$. The colloids were stable for weeks and nanoparticles are well dispersed when deposited on the substrate. It shows that nanoparticles in the hydrosols are efficiently electrostatically stabilized. The mean sizes of the nanoparticles were determined by image analysis of TEM pictures as $10 \mathrm{~nm}$ and $15 \mathrm{~nm}$ for $\mathrm{Ag}$ and $\mathrm{Au}$ nanoparticles, respectively.

$U V$-Vis optical absorption spectrum of a pure PMHT-Br polymer solution in DMSO/water $(1: 100, \mathrm{v} / \mathrm{v})$ mixture is shown in Fig. 3, curve a. The pure polymer is characterized by its well developed absorption band with a maximum at $423 \mathrm{~nm}$, which results from the $\pi \rightarrow \pi^{*}$ transition of the conjugated polythiophene backbone. The UV-Vis extinction spectrum (involves absorption and scattering of the incident light) of the $\mathrm{Ag}$ 
hydrosol shows a strong extinction band with a maximum at $395 \mathrm{~nm}$, characteristic for the surface plasmon resonance extinction (SPE) of isolated spherical Ag nanoparticles (Fig. 3, curve b) with the diameter below $30 \mathrm{~nm}$, embedded in water environment [8,18]. Similarly, Fig. 3, curve c, shows the UV-Vis extinction spectrum of the prepared gold hydrosol, with a strong SPE band centered at $520 \mathrm{~nm}$, which is typical for isolated spherical gold nanoparticles [17] dispersed in water.

Figs. 4 and 5 show the UV-Vis extinction spectra of Ag and Au hydrosols respectively just after the addition of PMHT-Br polymer dissolved in DMSO/water mixed (1:100) solvent at various concentrations. Upon addition of the PMHT-Br into the colloids immediate pronounced color changes were observed at certain concentration range of the added polymer.

At the lowest concentrations of the polymer admixed into the Ag hydrosol as $c_{\text {pol }}=5 \cdot 10^{-8}$ $M$ the SPE spectrum of the hydrosol remains almost unchanged, only a small decrease in the absorbance was observed. When increasing the concentration of the added polymer solution to $c_{\mathrm{pol}}=5 \cdot 10^{-7} \mathrm{M}$, (Fig. 4 curve b), the formation of a new plasmon band was observed on the long wavelength edge of the Ag hydrosol SPE band. With further increasing the polymer concentration this band becomes broader and shifts towards longer wavelengths, reaching a maximum absorbance for the polymer concentration $c_{\mathrm{pol}}=1 \cdot 10^{-6} \mathrm{M}$, with extinction maximum at $512 \mathrm{~nm}$ (Fig. 4 curve c). At this concentration, the sol becomes unstable with a tendency to fast aggregation. When the concentration of the added polymer exceeds the critical $c_{\text {pol }}$ value, the mixed sol becomes stable again and the long wavelength extinction band decreases, finally observed only as a broadening of the main SPE band of the parent hydrosol (Fig. 4, curves d - e).

It should be noted that the absorbance of the pure polymer solutions with the same concentrations for which the most pronounced spectral changes were observed is negligible compared to the SPE of the nanoparticles in the hydrosol and it cannot substantially contribute to the overall optical absorbance of the mixture. It means that the spectral changes upon addition of the polymer to the nanoparticles in the hydrosol can be assigned only to the processes and structure changes affecting the surface plasmon band of the metal nanoparticles. Only at the highest polymer concentration used within the experiment a small red shift of the maximum of the SPE band of Ag nanoparticles together with the band broadening were observed, which can be explained by its overlap with the pure polymer optical absorption.

Similar behavior was observed when adding the PMHT-Br polymer to the hydrosol containing Au nanoparticles with the following differences (see Figure 5): the SPE band of the pure hydrosol is centered at $524 \mathrm{~nm}$, the new absorption band upon the addition of the polymer solution appears at longer wavelengths, it reaches its maximum at $\lambda=684 \mathrm{~nm}$ at higher polymer concentrations $c_{p o l}=5 \cdot 10^{-6}$ and it is more pronounced (see Fig. 5 , curve d). In addition to these spectral changes, another band with a peak wavelength $\lambda=423 \mathrm{~nm}$ also appeared at higher polymer concentration $\left(c_{\mathrm{pol}}=1 \cdot 10^{-4} \mathrm{M}\right)$ that can be assigned to the absorption of conjugated polymer PMHT-Br.

The appearance of the second extinction band in the long wavelength region of the SPE spectrum is an indicator of an increased mutual interaction of plasmons localized within individual nanoparticles and it should be explained by a formation of nanoparticle aggregates [19]. The system under study contains two oppositely charged species: (i) $\mathrm{Ag}$ or $\mathrm{Au}$ nanoparticles that are negatively charged due to the presence of surface ions, as confirmed by the zeta potential measurements, and (ii) positively charged N-methylimidazolium side groups of the polymer. Hence, we can expect that the cationic polythiophene is effectively adsorbed at the NP surface due to the electrostatic attractive forces and it compensates the surface charge of the nanoparticles. As a result the nanoparticles aggregate, which is manifested by their characteristic optical spectra and fast sedimentation from the solvent. In particular, in the 
case of the Ag/PMHT-Br sol system with the critical polymer concentration $c_{\mathrm{pol}}=1 \cdot 10^{-6} \mathrm{M}$, and of the Au/PMHT-Br system with $c_{\mathrm{pol}}=5 \cdot 10^{-6} \mathrm{M}$, respectively, the amount of positively charged $\mathrm{N}$-methylimidazolium groups of the polymer present in the solution allows for the full coverage of the nanoparticle surface by the polymer. Consequently, it allows for the charge compensation resulting in a fast aggregation of nanoparticles into large assemblies manifested by the presence of two SPE bands in their extinction spectra (Fig. 4, curve c, and Fig. 5, curve d). The higher critical concentration observed for hydrosols with $\mathrm{Au}$ nanoparticles compared to the silver ones can be easily explained by the larger surface of $\mathrm{Au}$ nanoparticles. At higher cationic polymer concentrations, the excess of the positive charge prevents the nanoparticles from their aggregation. This explanation is confirmed by both the increased hydrosol stability for the system with the $1 \cdot 10^{-4} \mathrm{M}$ polymer concentration, and by the SPE spectra exhibiting a single broad extinction band red-shifted from that of the parent hydrosol.

SERS spectra of the Ag/PMHT-Br and Au/PMHT-Br sol systems normalized to the DMSO signal are shown in Fig. 6a and 6b. The samples were excited by the laser lines that are out of the PMHT-Br polymer resonance and closest to the respective position of the long wavelength SPE maxima, i.e. at $514.5 \mathrm{~nm}$ and $647.1 \mathrm{~nm}$, respectively, and Raman spectra were measured immediately after the system preparation as a function of polymer concentration.

The onset of SERS intensity enhancement appears at $\mathrm{c}_{\mathrm{pol}}=5 \cdot 10^{-8} \mathrm{M}$ and increases up to the critical concentration $1 \cdot 10^{-6} \mathrm{M}$ (for Ag NPs) or $5 \cdot 10^{-6} \mathrm{M}$ (for Au NPs) and then slightly decreases for higher concentrations. For the critical concentration the substantial increase of the SERS intensity is correlated with the appearance of the second SPE band in the extinction spectra. Strong correlation between the occurrence of the nanoparticles aggregates, optical spectra and SERS activity has previously been reported for the metal nanoparticles in colloidal systems and explained by the adsorbate induced particle aggregation [7]. In our experiments the highest Raman signals were clearly obtained for the systems containing large nanoparticles aggregates, in which a localization of polymer segments in the hot spots can be expected and in which the optical excitation closely matches the extinction band of the aggregates (512 nm or $684 \mathrm{~nm}$, for $\mathrm{Ag}$ and $\mathrm{Au}$ nanoparticles, respectively) resulting in the strongest enhancement of Raman scattering by an electromagnetic mechanism [20,21]. In the systems containing higher than critical concentration of the polymer the decrease of SERS intensity can be explained by weakening of the localized optical field between the nanoparticles caused by the increased interparticle spacing in the loosely packed metal nanoparticles/polymer aggregates, as well as by the poor matching between the excitation laser lines and maximum of SPE bands.

The SERS spectrum of the cationic polythiophene is dominated by the intense band at $1463 \mathrm{~cm}^{-1}$, characteristic to the $\mathrm{C}_{\alpha}=\mathrm{C}_{\beta}$ bond of the thiophene ring. This band was used for the evaluation of the SERS enhancement factor. The SERS enhancement factor in these composite systems was at least $10^{4}$, as obtained from the comparison of the Raman signals of the polymer solution and of the composite sols with the same polymer concentration to the reference DMSO signal.

Optical properties of Ag/PTAA sol systems were also studied for the comparison. The absorption spectrum of the anionic PTAA polymer in a DMSO/water (1:100) solution shows its maximum at $417 \mathrm{~nm}$ (Fig.7, curve a). Contrary to the cationic PMHT-Br there was no formation of the second SPE band observed in the extinction spectra upon the addition of the PTAA to the hydrosol at any concentration. Only a slight increase of the SPE band was observed due to the overlap of the polymer and Ag hydrosol absorption (Fig. 7, curve c). Similarly, no enhancement in the Raman signal occurred after mixing the Ag NPs with the PTAA (not shown here). It is obvious that in case of the anionic polymer, which contains 
$\mathrm{COO}^{-}$negatively charged side groups, adsorption of the polymer to the nanoparticle surface is not allowed due to the electrostatic repulsion and, hence, no aggregation is possible. As a result, no enhancement of the Raman scattering has been observed.

Layer by layer deposition of thin films: The electrostatic interactions that take place during the aggregation of sol systems were successfully utilized also for the preparation of PMHT$B r / A u-N P s / P T A A$ multilayered structures by layer-by-layer deposition technique. The optical spectra of such structure cast on a quartz substrate recorded between the consecutive immersions of the substrate into the aqueous solutions of the cationic PMHT-Br, Au hydrosol and anionic PTAA are shown in Fig. 8. The optical spectra confirm the progressive build-up of the layered structures. Apparently, the coverage of the deposited cationic layer by adsorbed $\mathrm{Au}$ nanoparticles is not complete, leaving some uncompensated cationic groups for the subsequent adsorption of the PTAA layer. As can be seen, the surface plasmon resonance of $\mathrm{Au}$ nanoparticles contributes markedly to the overall extinction of the composite. The SPE band of the first $\mathrm{Au}$ layer was observed at $536 \mathrm{~nm}$ that corresponds to weakly coupled $\mathrm{Au}$ nanoparticles arranged in 2-dimensional assembly. The observed red shift of the plasmon extinction maximum to $574 \mathrm{~nm}$ for the alternating polymers multilayer containing $7 \mathrm{Au}$ layers proves an increased electronic coupling of plasmons in the multilayer structure with increasing number of layers.

\section{Conclusion}

It has been shown that the aggregation of $\mathrm{Au}$ or $\mathrm{Ag}$ nanoparticles facilitated by their electrostatic interactions with cationic polythiophene provides systems containing assemblies of electronically coupled plasmonic nanoparticles. Such systems showed the formation of "hot spots" with a strong local electromagnetic field enhancement effects, evidenced by the enhanced Raman scattering (SERS). Maximum enhancement was achieved when the components ratio provided the charge balance between the nanoparticles and cationic polythiophene, at which the nanoparticles are nearly single-polymer-layer coated and a tight coupling of plasmons of adjacent nanoparticles is allowed. By subsequent adsorption of $\mathrm{Au}$ nanoparticles and polythiophenes with cationic and anionic side-groups the alternating multilayer structures were prepared using layer-by-layer deposition technique. These layers showed efficient coupling of plasmons of individual nanoparticles, evidenced by the shift of their plasmon resonance frequency.

\section{Acknowledgments}

Financial supports of the Academy of Sciences of the Czech Republic (grant KAN100500652) and of the Czech Science Foundation (project 203/07/0717) are greatly acknowledged. D. Bondarev is indebted to the Czech Science Foundation for the fellowship (project 203/08/H032). We also thank to Ms J. Hromadkova for performing TEM measurements. 


\section{References}

1. P.V. Kamat, J. Phys. Chem. B 106, 7729 (2002)

2. J. M. Laskar, D. Mohanta, A. Choudhury, Eur. Phys. J. Appl. Phys. 46 , 20602 (2009)

3. P.K. Jain, X. Huang, I.H. El-Sayed, M.A. El-Sayed, Plasmonics, 2, 107(2007)

4. T. He, P. Li, Z. Cai, Y. Mo, Eur. Phys. J. Appl. Phys. 38 , 15 (2007)

5. Y.Wang, S.Guo, H.Chen, E.Wang, J.Colloid Interf.Sci. 318, 82 (2008)

6. W. L. Leong, P.S. Lee, S. G. Mhaisalkar, T. P. Chen, A. Dodabalapur. App. Phys. Lett. 90, 042906 (2007)

7. J. A. Creighton, C. G. Blatchford, M. G. Albrecht, J. Chem. Soc. Faraday Trans. 75, 790 (1979)

8. J.A. Creighton, in: R. Chang, T. Furtak (Eds.), Surface Enhanced Raman Scattering, Plenum Press, New York, (1982) p. 315

9. M. I. Stockman, V. M. Shalaev, M. Moskovits, R.Botet, T. F. George, Phys. Rev 46, 2821(1992)

10. L.H. Chen, S. Xu, D. McBranch, D. Whitten J Am Chem Soc 122, 9302 (2000)

11. P.V. Kamat, J. Phys. Chem. C 111, 2834 (2007)

12. O. Dammer, B. Vlckova, M. Prochazka, J. Sedlacek, J. Vohlidal, and J. Pfleger, Phys. Chem. Chem. Phys 11, 5455 (2009)

13. D.Bondarev, J. Zedník, I. Šloufová, A. Sharf, M. Prochazka, J. Pfleger, J. Vohlídal. J. Polym. Sci. Polym. Chem. 48, 3073 (2010)

14. B.S. Kim, L. Chen, J. Gong, Y. Osada, Macromolecules 32, 3964(1999)

15. B.Vlčková, P.Matějka, J.Šimonová, K.Čermáková, P.Pančoška, V.Baumruk, J. Phys. Chem. 97, 9719 (1993)

16. P.Šmejkal, B.Vlčková, M.Procházka, P.Mojzeš, J. Pfleger, J. Vibr. Spectrosc. 19, 243(1999)

17. P.C. Lee, D. Meisel, J. Phys. Chem. 86, 3391(1982)

18. A.A. Ashkarran, A. Iraji zad, M.M. Ahadian, M.R. Hormozi Nezhad Eur. Phys. J. Appl. Phys. 48, 10601 (2009)

19. J.J. Storhoff, A.A. Lazarides, R.C. Mucic, C.A. Mirkin, R.L. Letsinger, G.C. Schatz. J. Am. Chem. Soc. 122, 4640(2000)

20. H. Xu, J. Aizpurua, M. Kall, P. Apell, Phys. Rev. 62, 4318(2000) 10903(1999)

21. V. A. Markel, V. M. Shalaev, P. Zhang, W. Huynh, L. Tay, T.L. Haslet, M. Moskovits, Phys. Rev. 59, 10903(1999) 


\section{Figure Captions}

Fig. 1. Chemical structures of a) cationic polythiophene, poly\{3-[6-(1-methylimidazolium-3yl)hexyl]thiophene-2,5-diyl bromides, PMHT-Br, and b) anionic polythiophene, poly[(thiophene-3-yl)acetic acid], PTAA.

Fig. 2. TEM images of a) borate stabilized Ag nanoparticles, b) citrate stabilized $\mathrm{Au}$ nanoparticles.

Fig. 3. Curve a: UV-Vis optical absorption spectrum of the pure polymer PMHT-Br $(c$ pol $=$ $\left.1 \cdot 10^{-5} \mathrm{M}\right)$ in $\mathrm{DMSO} /$ water $(1: 100 \mathrm{v} / \mathrm{v})$ mixed solvent, curve $\mathrm{b}$ : surface plasmon extinction (SPE) spectrum of the parent Ag colloid, curve c: SPE spectrum of the parent Au colloid.

Fig. 4. Extinction spectrum of the parent Ag colloid (curve a), and extinction spectra of $\mathrm{Ag}$ colloid with various concentrations of added PMHT-Br polymer: $5 \cdot 10^{-7} \mathrm{M}$ (curve b), $1 \cdot 10^{-6} \mathrm{M}$ (curve c), $1 \cdot 10^{-5} \mathrm{M}$ (curve d) and $1 \cdot 10^{-4} \mathrm{M}$ (curve e).

Fig. 5. Extinction spectrum of the parent Au colloid (curve a), and extinction spectra of $\mathrm{Au}$ colloid with various concentrations of added PMHT-Br polymer: $5 \cdot 10^{-7} \mathrm{M}$ (curve b), $1 \cdot 10^{-6} \mathrm{M}$ (curve c), $5 \cdot 10^{-6} \mathrm{M}$ (curve d) and $1 \cdot 10^{-4} \mathrm{M}$ (curve e).

Fig. 6. SERS spectra hydrosols with various concentrations of added PMHT-Br polymer: a) Ag hydrosol, excitation wavelength $514.5 \mathrm{~nm}, \mathrm{~b})$ Au hydrosol, excitation wavelength $647 \mathrm{~nm}$. Concentrations of the polymer shown in the figure.

Fig. 7. UV-Vis optical absorption spectrum of pure PTAA polymer $\left(1.0 \cdot 10^{-5} \mathrm{M}\right)$ in DMSO/ water (1:100) mixed solvent (curve a), SPE spectrum of the parent Ag hydrosol (curve b) and SPE spectrum of silver hydrosol upon addition of PTAA polymer at concentration $1 \cdot 10^{-5} \mathrm{M}$ (curve c).

Fig. 8. Increase of the optical extinction of a PMHT-Br/Au/PTAA multilayer structure during the layer-by-layer deposition. The concentration of both polymer solutions used for the deposition was $10^{-5} \mathrm{M}$. The shift of the maximum of the SPE of Au nanoparticles is marked. 


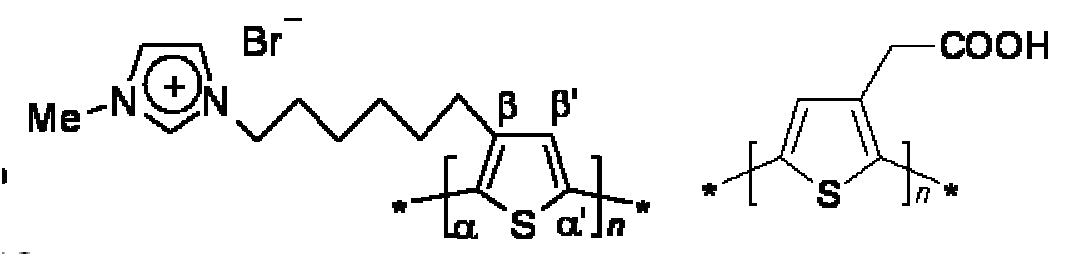
(a) PMHT-Br
(b) PTAA

Fig. 1 


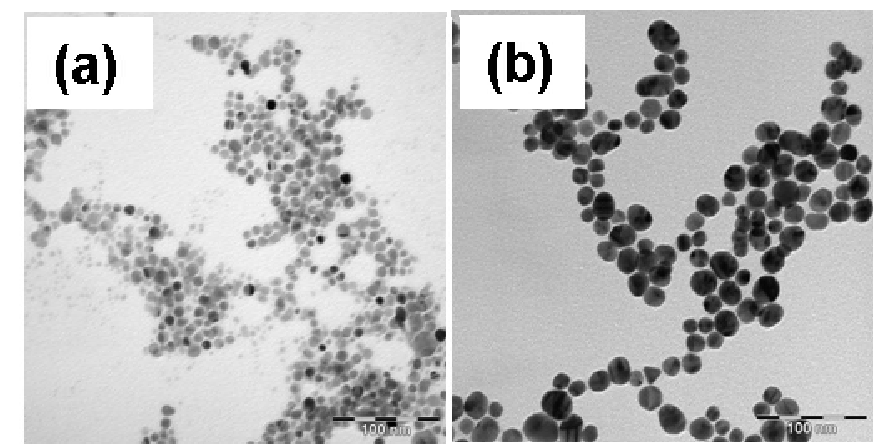

Fig. 2 
1. 1

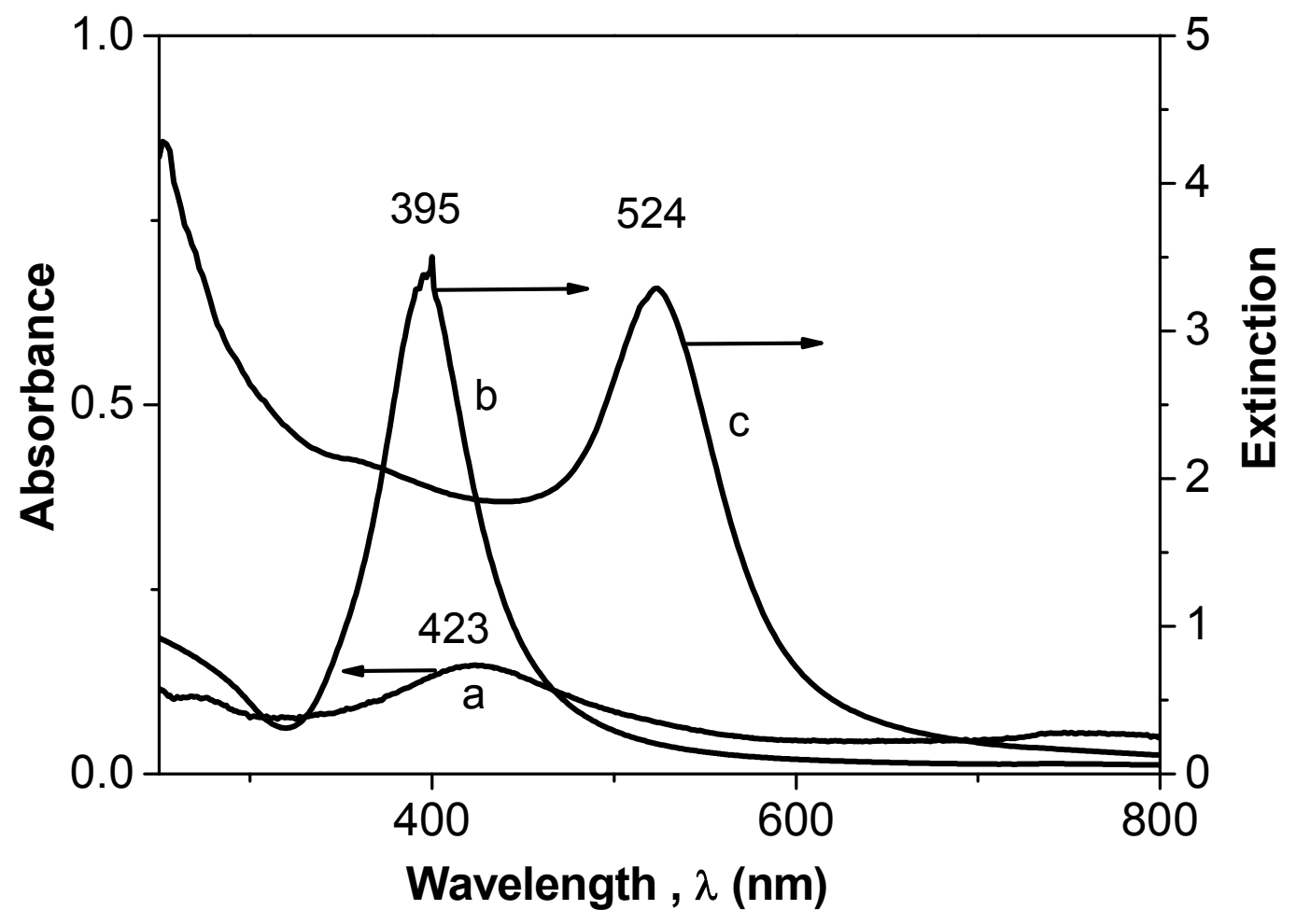

Fig. 3 


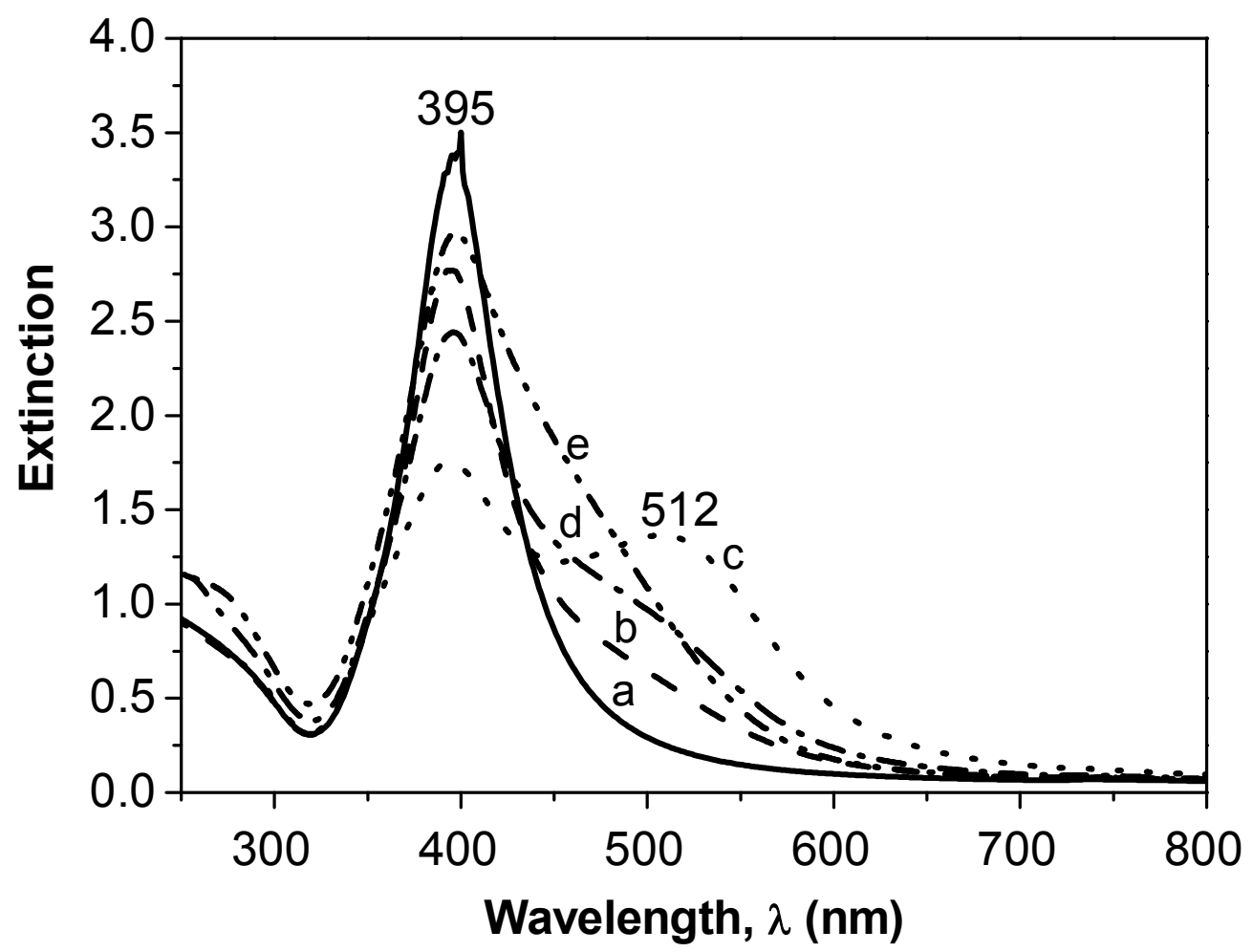

Fig. 4 


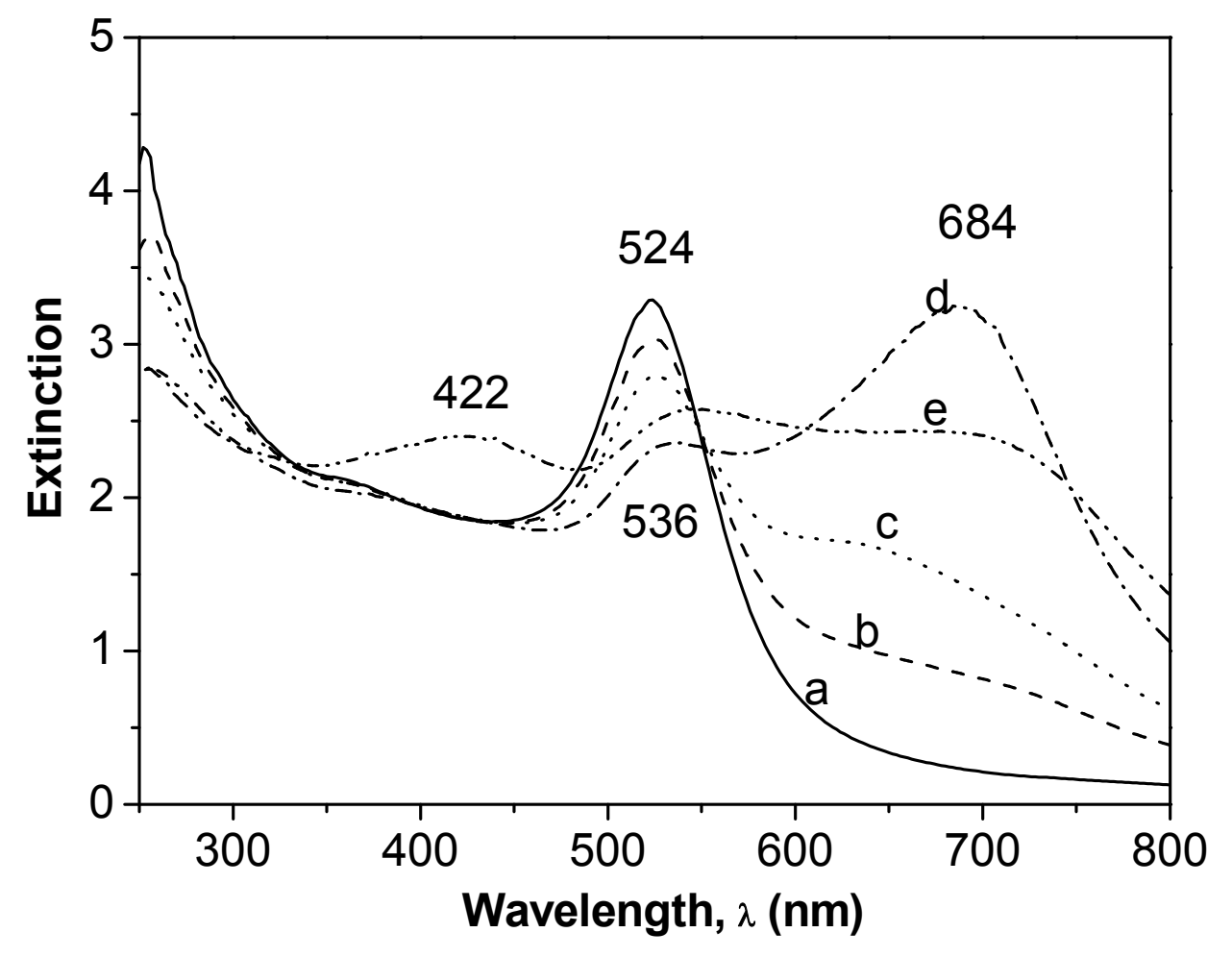

Fig. 5 


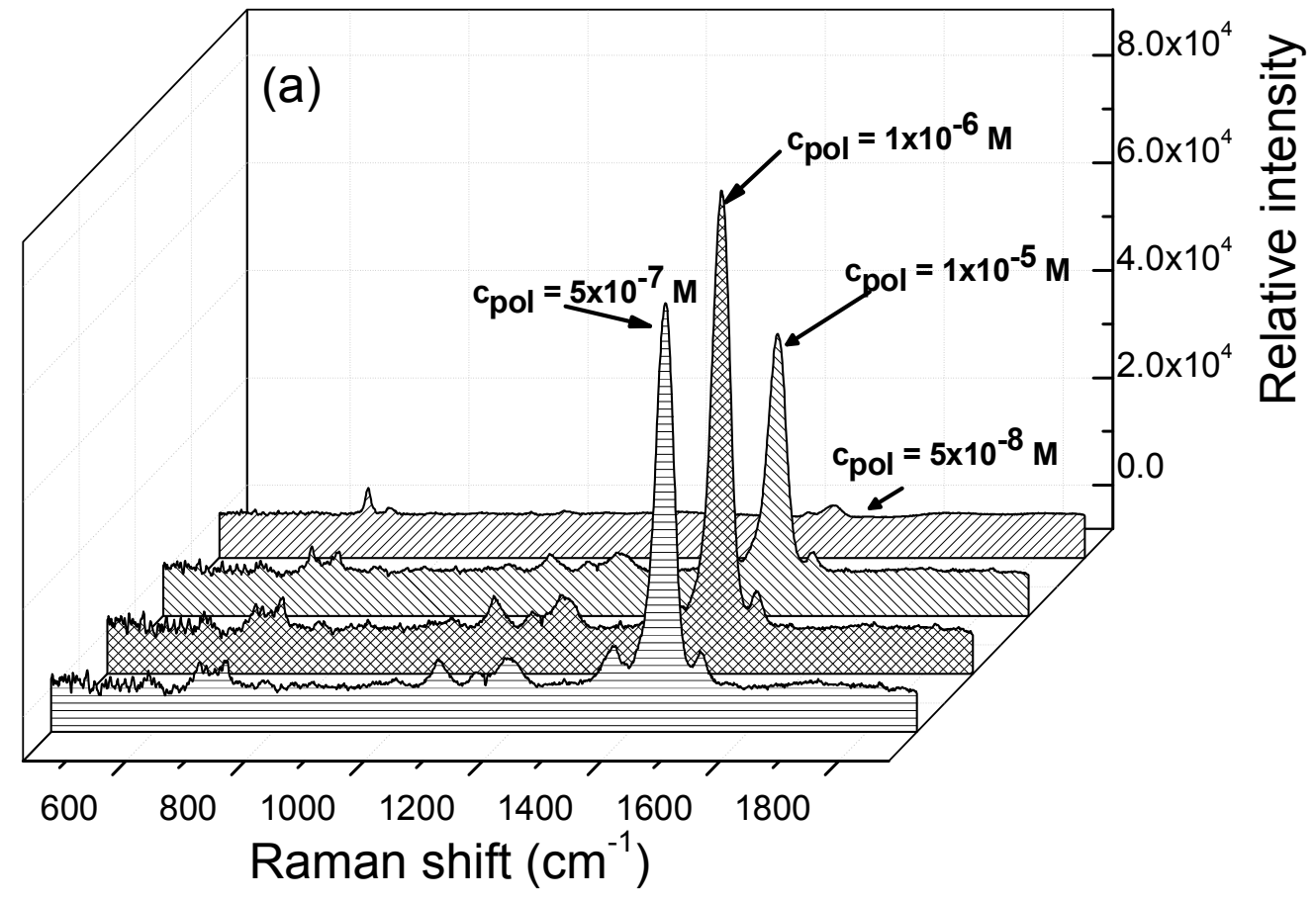

Fig. 6(a) 


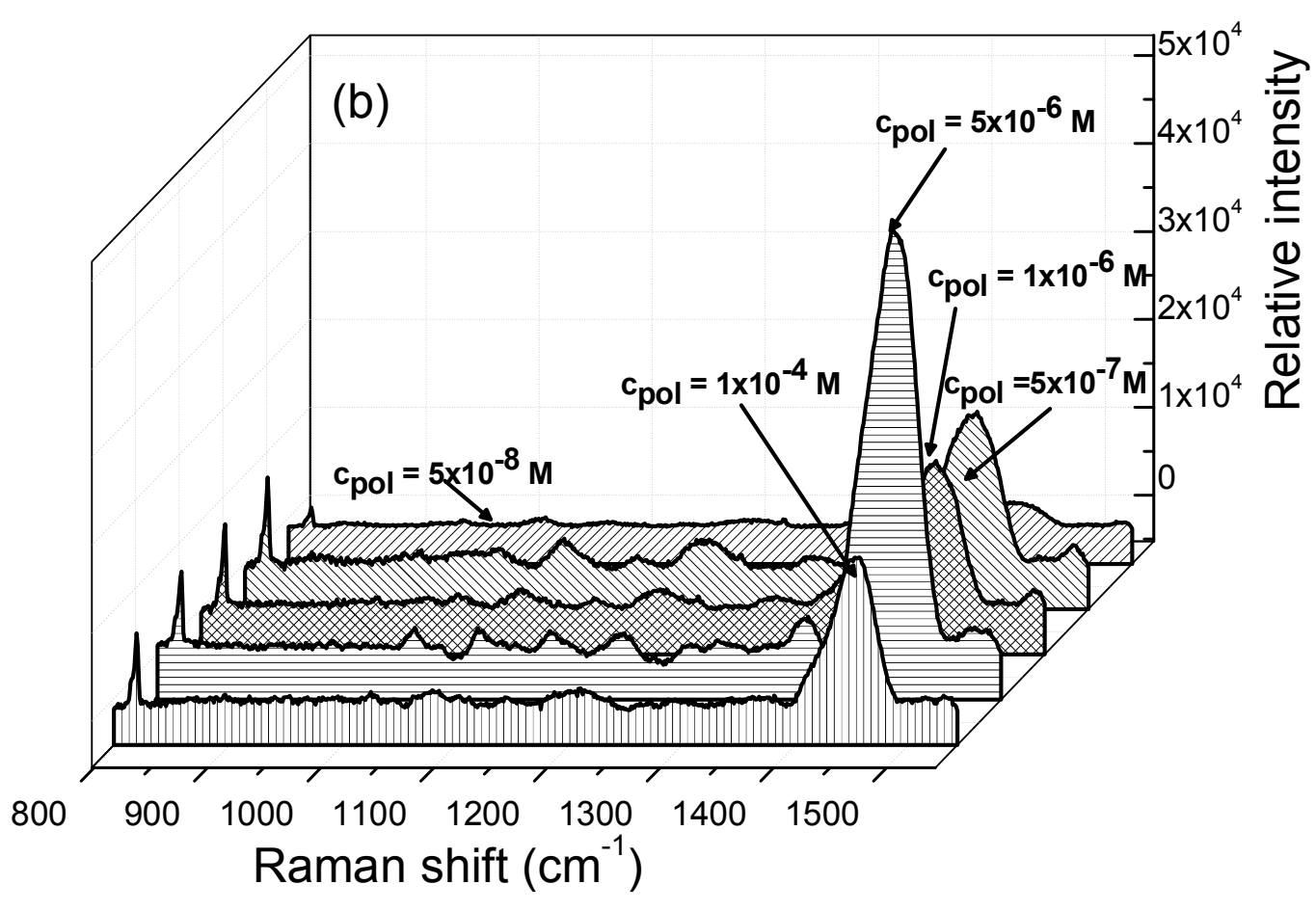

Fig. 6 (b) 


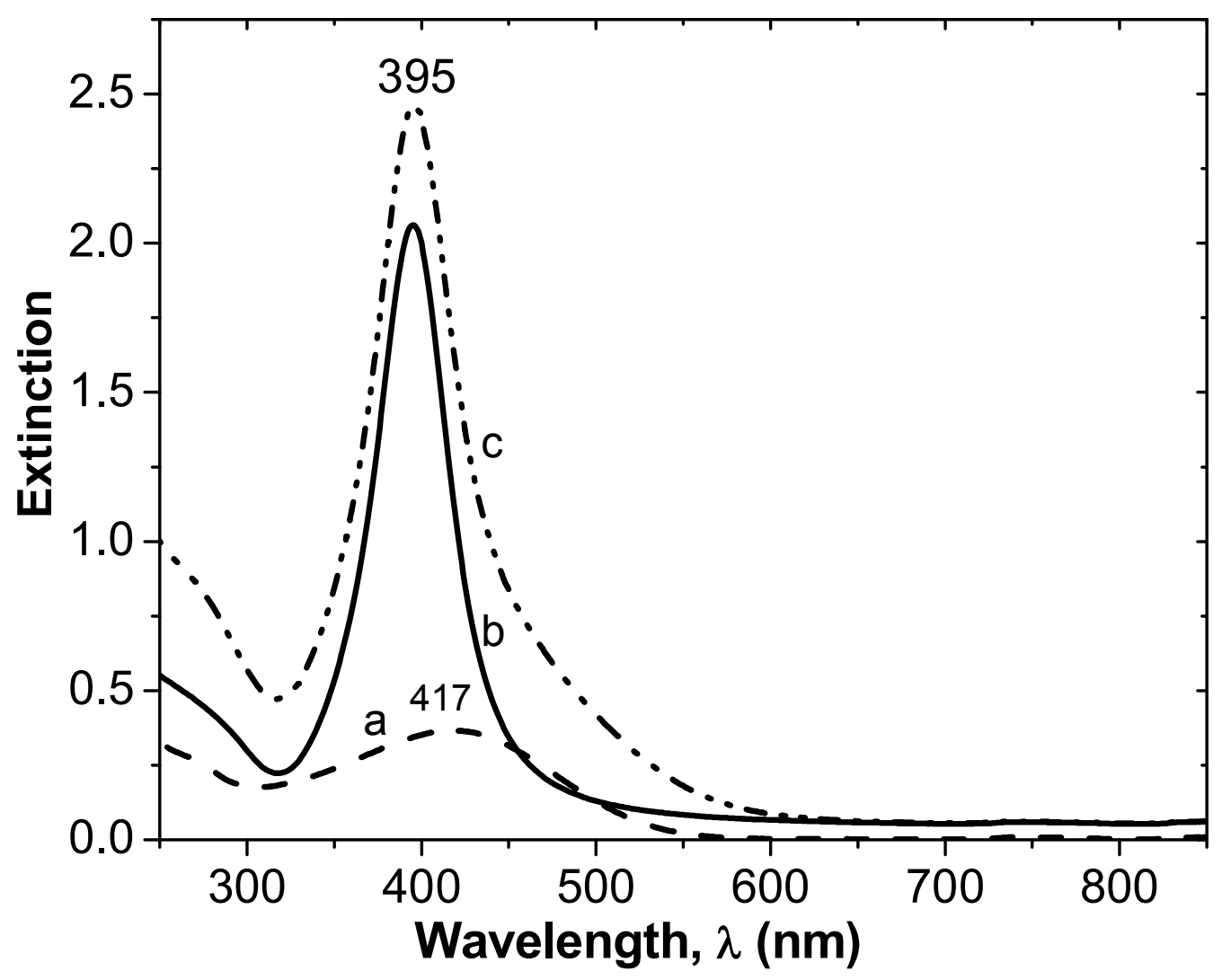

Fig. 7 


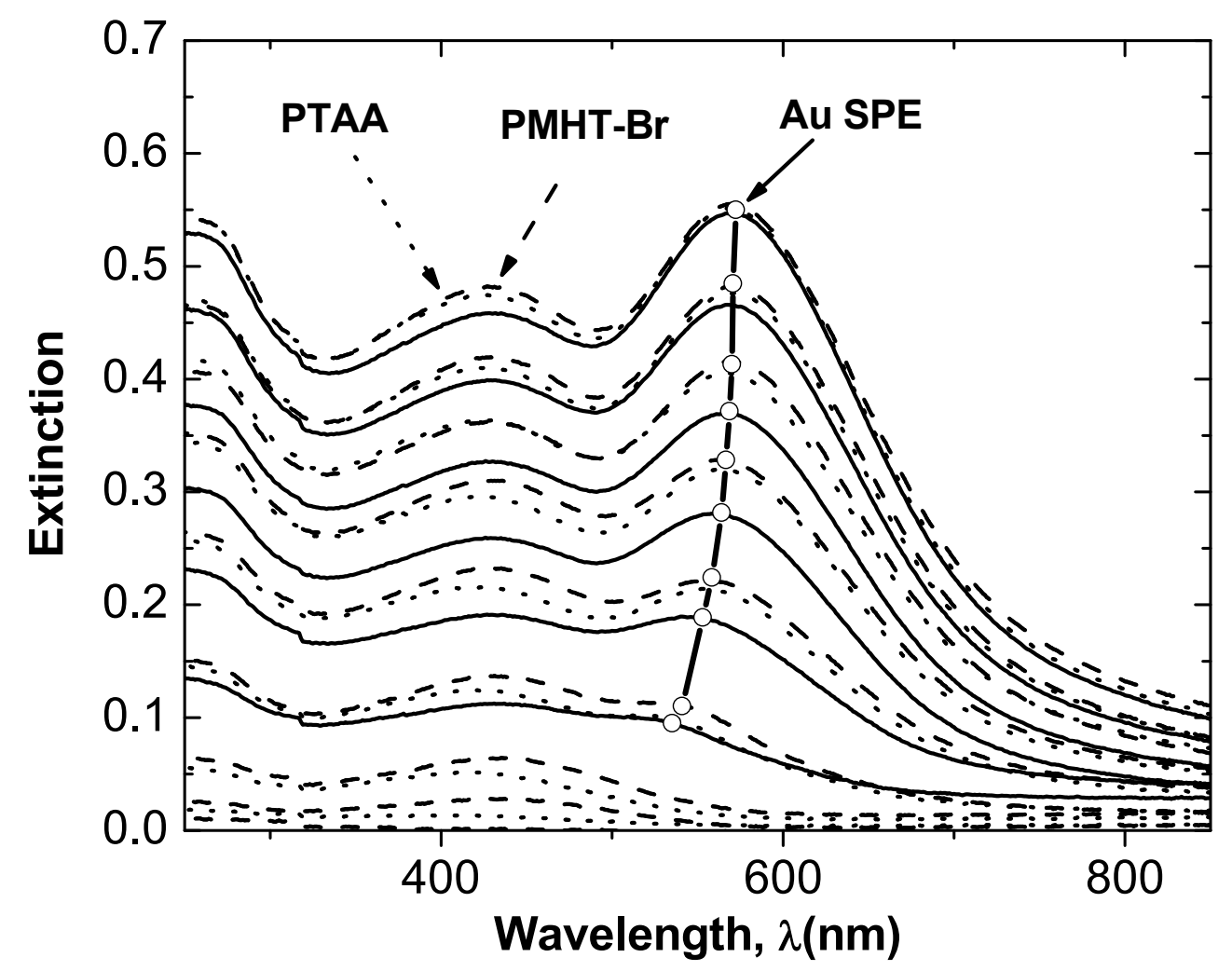

Fig. 8 